\title{
Radiation From a Plasma-Clad Axially-Slotted Cylinder
}

\author{
W. V. T. Rusch \\ Contributions from Electrical Engineering Department, University of Southern California, \\ Los Angeles 7, Calif.
}

(Received October 9, 1962)

\begin{abstract}
Expressions have been developed for the complete radiation pattern from a finite axial slot on an infinite circular cylinder covered with a homogeneous, uniform plasma sheath. The resulting field expressions are functions of both the longitude angle, $\phi$, and the polar angle, $\theta$. Numerical results have been obtained for a thin lossless plasma to determine the variation of the field pattern with plasma frequency, plasma thickness, slot width, and cylinder radius. These results are presented graphically to illustrate various properties of the radiating system.
\end{abstract}

\section{Introduction}

The theory of radiation from slotted-cylinder antenna systems has been known for many years [Wait, 1959, bibliography]. This theory is especially useful in predicting the requirements for communicating with high-speed cylindrically shaped space vehicles by means of slots on the body of the vehicle. However, it is known that upon the impact of re-entry into the earth's atmosphere, an ionized plasma sheath is formed around the vehicle. To a first approximation, such a plasma sheath may be represented by a uniform dielectric coating with an equivalent dielectric constant determined by the properties of the sheath. The slotted-cylinder radiation theory has been extended to include the effects of a uniform dielectric coating [Wait, 1959, ch. 16]. However, numerical results have only been obtained for the equatorial-plane radiation pattern.

Since it is desirable to know the complete radiation pattern, expressions have been derived for the field of such a slotted, plasma-clad cylindrical radiator for all values of $\theta$ and $\phi$ which meet the far-zone conditions. For the purpose of numerical simplicity, results have been obtained only for the case of a lossless sheath which is thin relative to a wavelength. The calculations could also have been extended to lossy plasmas with only slight modinications. 'The numerical results are presented to demonstrate the effect upon the radiation pattern of the various parameters of the radiating system. Of special interest is the influence of the plasma frequency/ operating frequency ratio, since cutoff effects cause serious problems in communicating with re-entry vehicles.

1 This research was sponsored by the Air Force Cambridge Research Labo ratories of the Office of Aerospace Research under contract A F 19(604)-6195.

\section{Derivation of the Radiation Fields}

An infinite, perfectly conducting circular cylinder of radius $a$ is coaxial with the $z$-axis (fig. 1 ). The cylinder is covered by a homogeneous, isotropic

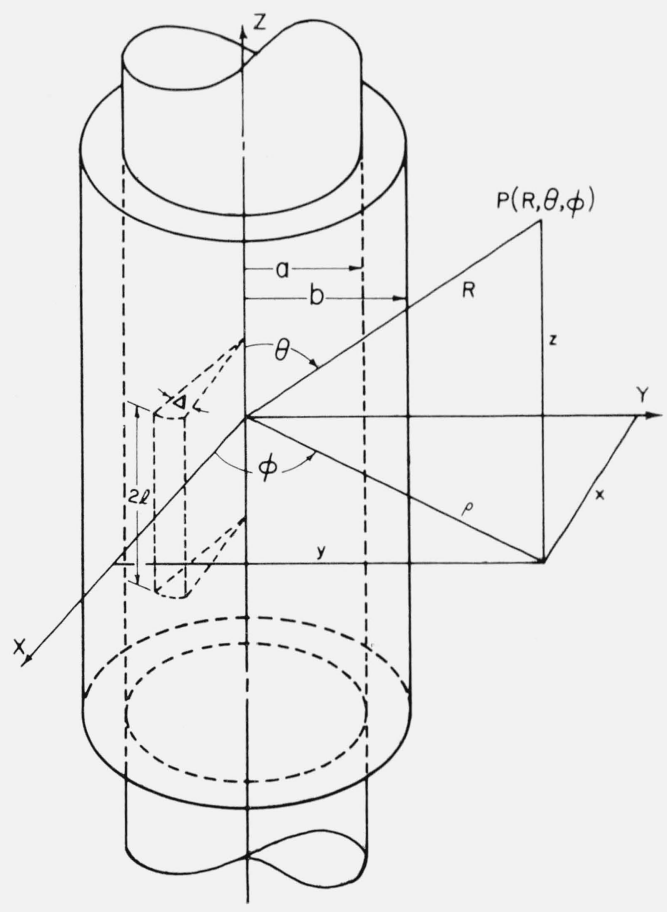

Figure 1. Geometry of slotted cylinder with plasma sheath. 
plasma coating of permittivity $\epsilon(\omega)=\epsilon_{0} \cdot\left[1-\omega_{p}^{2} / \omega^{2}\right]$ and permeability $\mu$ extending from $a \leq \rho \leq b$. An axial slot on the surface of the cylinder $(\rho=a)$ extends from $-l \leq z \leq+l$ and $-\frac{\Delta}{2} \leq \phi \leq+\frac{\Delta}{2}$. Because of the assumed nature of the fields in the slot, the tangential electric fields at $\rho=a$ are:

$$
\begin{gathered}
E_{z}(a, \phi, z)=0 \\
E_{\phi}(a, \phi, z)=\left\{\begin{array}{l}
0,|z|>l \text { or }|\phi|>\frac{\Delta}{2} \\
E_{0}(\phi, z),|z| \leq l \text { and }|\phi| \leq \frac{\Delta}{2} .
\end{array}\right.
\end{gathered}
$$

The tangential electric field at $\rho=$ a may be expanded:

$$
E_{\phi}(a, \phi, z)=\sum_{n=-\infty}^{+\infty} e^{i n \phi} \int_{-\infty}^{+\infty} e_{n}(h) e^{i n z} d h
$$

where

$$
\begin{gathered}
e_{n}(h)=\frac{1}{(2 \pi)^{2}} \int_{-\infty}^{+\infty} d z \int_{-\pi}^{+\pi} d \phi E_{\phi}(a, \phi, z) e^{-i n \phi} e^{-i h z} \\
=\frac{1}{(2 \pi)^{2}} \int_{-l}^{+l} d z \int_{-\Delta / 2}^{+\Delta / 2} d \phi E_{0}(\phi, z) e^{-i n \phi} e^{-i h z} .
\end{gathered}
$$

Following the analysis of Wait, the total electromagnetic field may then be described in terms of an electric Hertz vector and a magnetic Hertz vector [Stratton, 1941]. Then, using a standard saddlepoint technique [Wait, 1959] and assuming that $\left(\frac{b-a}{\lambda}\right)<<1$ and $\omega$ not appreciably less than $\omega_{p}$, it can be shown that in the far zone of a half-wave slot:

$E_{\phi}(R, \theta, \phi)=\left\{\frac{V_{0} e^{i k_{0} R}}{\pi^{2} B R}\right\} \cos \left(\frac{\pi}{2} \cos \theta\right) \sum_{n=0}^{\infty} \frac{\epsilon_{n} J_{0}\left(\frac{n \Delta}{2}\right)\left\{D\left(\cos ^{2} \theta-\beta^{2}\right) H_{n}^{\prime}(B \sin \theta)+\beta^{2} \sin \theta H_{n}(B \sin \theta)\right\} e^{-i \frac{n \pi}{2}} \cos n \phi}{\left\{Q\left[H_{n}^{\prime}(B \sin \theta)\right]^{2}+R_{n}\left[H_{n}^{\prime}(B \sin \theta)\right]\left[H_{n}(B \sin \theta)\right]+S_{n}\left[H_{n}(B \sin \theta)\right]^{2}\right\}}$

where $\epsilon_{n}=1$ if $n=0, \epsilon_{n}=2$ if $n=1,2,3 \ldots$

$$
\begin{aligned}
D & =k_{0}(b-a) \\
\beta^{2} & =1-\omega_{p}^{2} / \omega^{2}=k^{2} / k_{0}^{2} \\
B & =k_{0} b \cong k_{0} a \\
Q & =D \sin ^{2} \theta\left(\cos ^{2} \theta-\beta^{2}\right) \\
R_{n} & =\sin ^{3} \theta\left[\beta^{2}\left(1-D^{2}\right)+D^{2}\left(\cos ^{2} \theta+\frac{m^{2}}{B^{2}}\right)\right] \\
S_{n} & =D\left[\beta^{2} \sin ^{4} \theta+\frac{n^{2}}{B^{2}}\left(\beta^{2} \cos ^{2} \theta-1\right)\right]
\end{aligned}
$$

$H_{n}(B \sin \theta)=J_{n}(B \sin \theta)+i N_{n}(B \sin \theta)$.

Equation (5) easily yields results for two special cases:

Equatorial plane fields: When $\theta=\pi / 2$, (5) reduces to

$$
\begin{aligned}
E_{\phi}\left(R, \frac{\pi}{2}, \phi\right)=\left\{\frac{V_{0} e^{i k_{0} R}}{\pi^{2} B R}\right\} \sum_{n=0}^{\infty} \\
\\
\frac{\epsilon_{n} J_{0}\left(\frac{n \Delta}{2}\right) \cos n \phi e^{-i \frac{n \pi}{2}}}{H_{n}^{\prime}(B)+D\left(1-\frac{n^{2}}{\beta^{2} B^{2}}\right) H_{n}(B)} .
\end{aligned}
$$

Cylinder in free space: If $D=0$ or $\beta=1$, (6) reduces to $E_{\phi}\left(R, \frac{\pi}{2}, \phi\right)=\left\{\frac{V_{0} e^{i k_{0} R}}{\pi^{2} B R}\right\} \sum_{n=0}^{\infty} \frac{\epsilon_{n} J_{0}\left(\frac{n \Delta}{2}\right) \cos n \phi e^{-i \frac{n \pi}{2}}}{H_{n}^{\prime}(B)} \cdot($
The field in the half-wave slot has been assumed to be

$$
E_{0}(\phi, z)=\frac{V_{0} \cos k_{0} z}{a \pi \sqrt{\left(\frac{\Delta}{2}-\phi\right)\left(\frac{\Delta}{2}+\phi\right)}} .
$$

This field distribution may reasonably approximate the field in a narrow slot with knife edge sides such as might be found in a slotted waveguide or slotted cavity. If, however, the slot is fed by an open-ended waveguide in the $T E_{10}$ mode, a more appropriate approximation would be

$$
E_{0}(\phi, z)=\frac{V_{0} \cos k_{0} z}{a \Delta} .
$$

Equation (8) leads to a term $J_{0}(n \Delta / 2)$ in the field expressions, and (9) leads to a term $\frac{\sin \frac{n \Delta}{2}}{\frac{n \Delta}{2}}$. Since the former may be expanded as $1-\frac{(n \Delta)^{2}}{16}+\ldots$ and the latter as $1-\frac{(n \Delta)^{2}}{24}+\ldots$, there is little significant difference in the field patterns for extremely small values of $\Delta$.

\section{Results of the Numerical Calculations}

The amplitude and phase lag of $E_{\phi}$ [from (5)] are plotted in polar form in figures 2 through 18 . The various parameters are $\omega / \omega_{p}$, a measure of the 
plasma density; $B$, the outer radius of the sheath in wave numbers; $D$, the thickness of the sheath in wave numbers; $\Delta$, the angular width of the slot in radians; $\theta$, the colatitude angle; $\phi$, the longitude angle. Each amplitude pattern is individually normalized. Consequently, direct comparison of relative amplitudes may not be made from one figure to another. Furthermore, each set of patterns assumes constant slot voltage, although the impedance of the slot may undergo extreme changes. The relative amplitude of these patterns will be entirely different if the slot is driven by a constant power source.
Various horizontal and vertical "cuts" in the amplitude pattern are plotted in figures 2 and 3 for relatively low plasma density $\left(\omega / \omega_{p}=3.162\right)$. The horizontal cuts (fig. 2) exhibit a broadside maximum with a standing wave pattern toward the rear of the cylinder, suggesting a trapped peripheral wave. The vertical patterns (fig. 3 ) also possess a broadside maximum. It is of interest to note that the pattern in the plane through the slot and the cylinder axis $(\phi=0)$ very closely resembles the corresponding pattern from a half-wave slot on an infinite plasmaclad conducting plane.

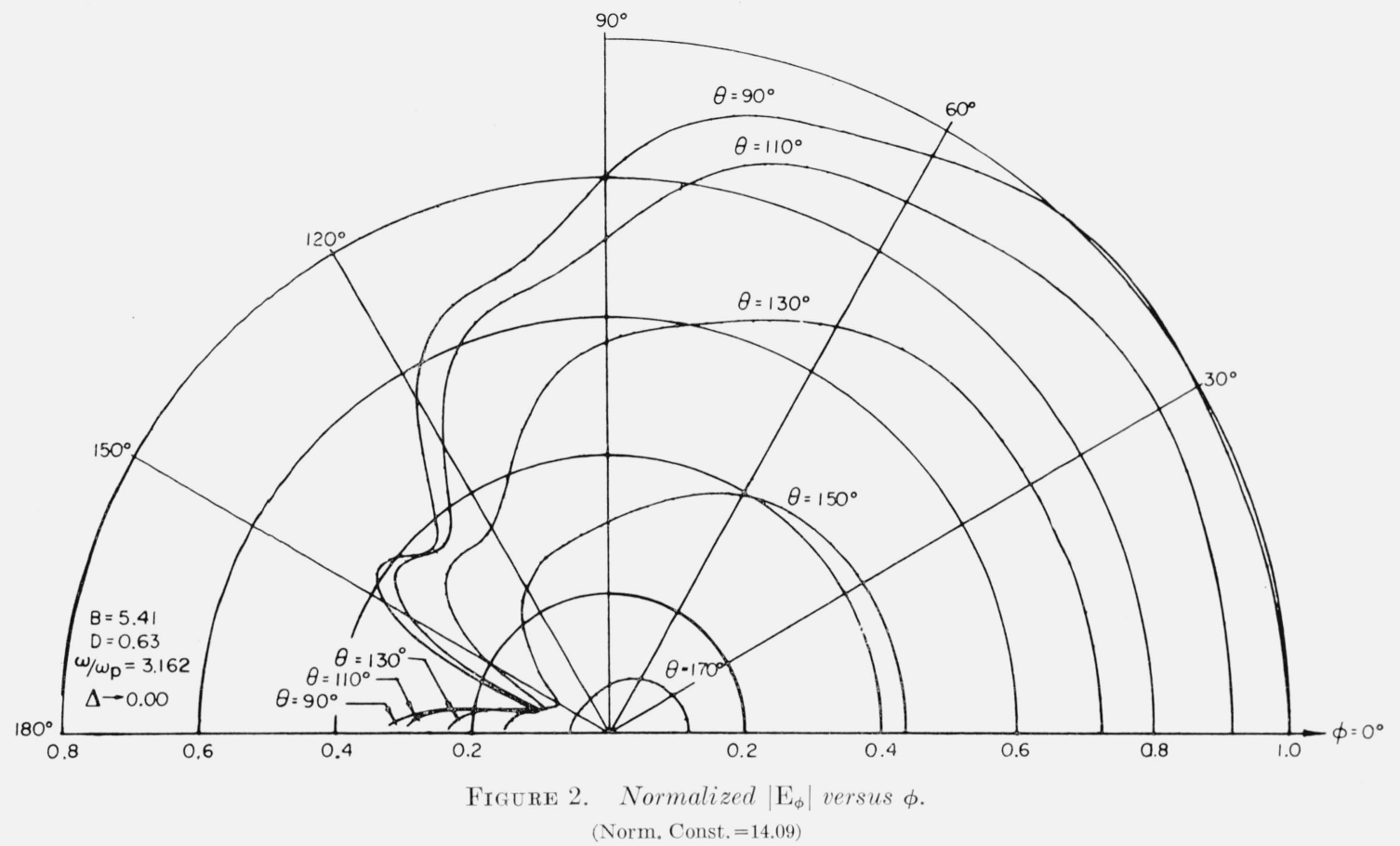

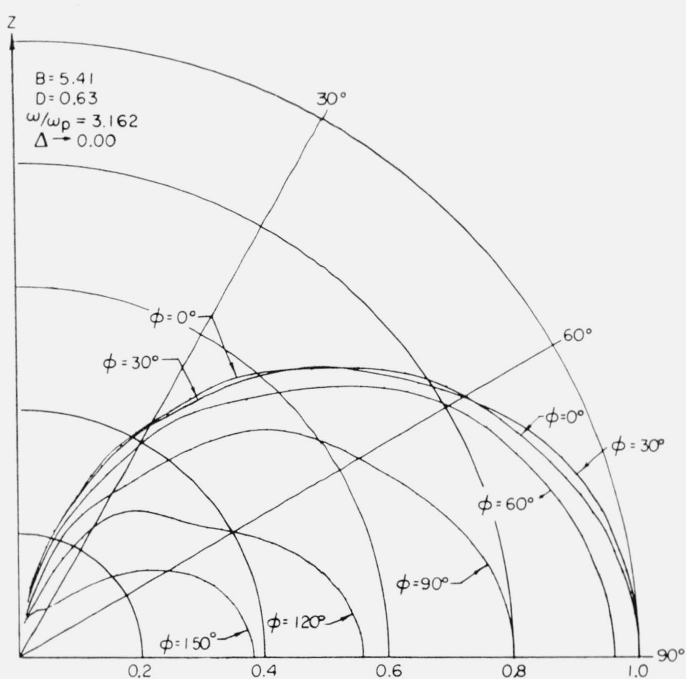

Figure 3. Normalized $\left|\mathrm{E}_{\phi}\right|$ versus $\theta$. (Norm. Const. $=14.09$ )
The same patterns are plotted in figures 4 and 5 for an increased plasma density $\left(\omega / \omega_{p}=1.05\right)$. The standing wave nature of the horizontal patterns is no longer distinguishable. In the forward direction $(\phi=0)$ a local maximum is beginning to appear above (and below, since symmetry exists about the equatorial plane) the equatorial plane. This local maximum resembles the pattern from the slotted plasmaclad plane where the first few dominant leaky waves contribute to off-broadside maxima in the far-field pattern. However, since the exact nature of the leaky-wave poles has not been determined for the plasma-clad cylinder, quantitative confirmation of the leaky-wave interpretation has not been made.

Figure 6 indicates the effect of varying the plasma density upon the equatorial plane field patterns. As $\omega$ passes below $\omega_{p}$, less energy is guided peripherally, and the pattern is more sharply broadside-directed. This result is to be expected, since propagating waves do not exist in the opaque plasma layer below plasma resonance, and energy "diffuses" through the sheath 


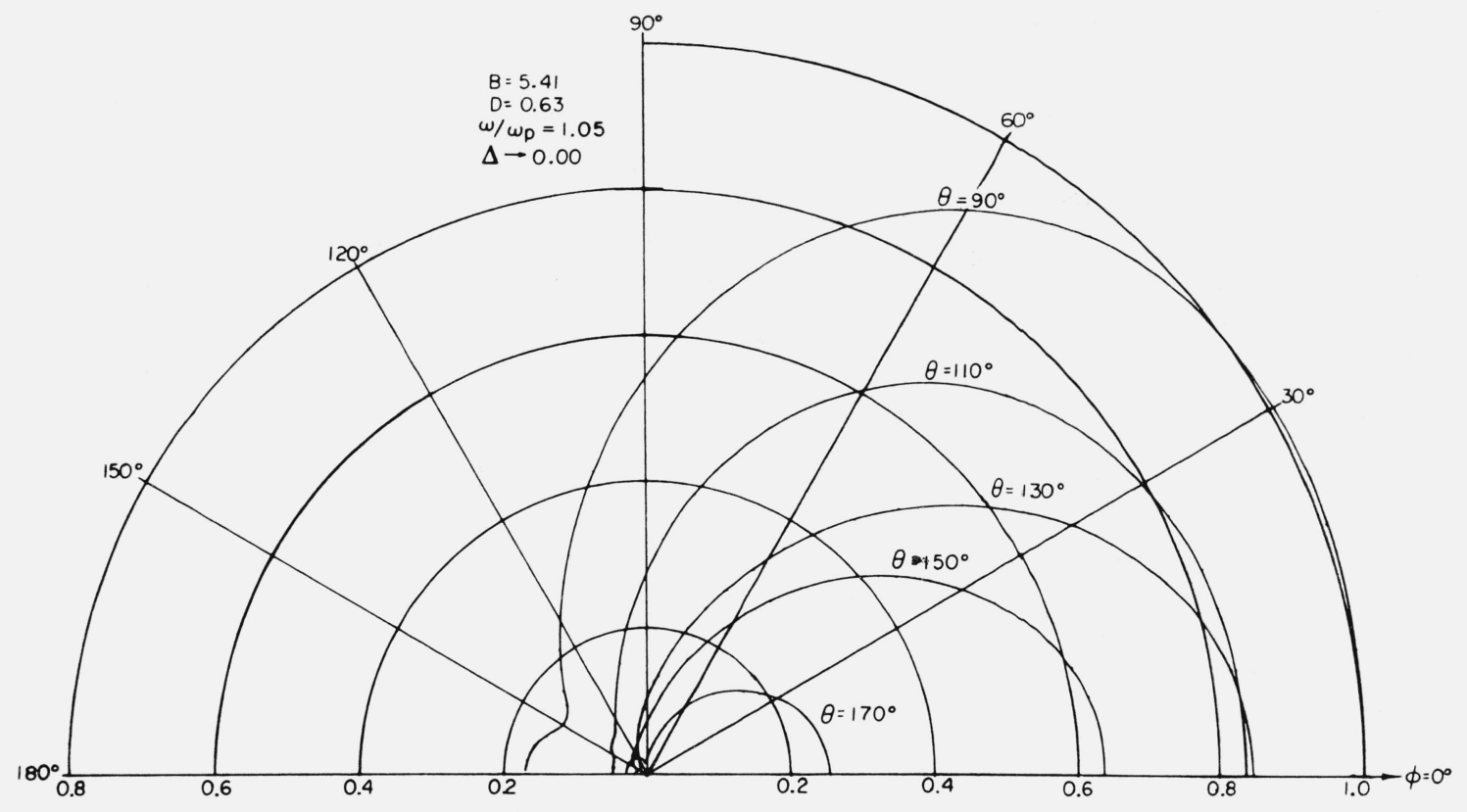

Figure 4. Normalized $\left|\mathrm{E}_{\phi}\right|$ versus $\phi$.

(Norm. Const. $=10.01$ )

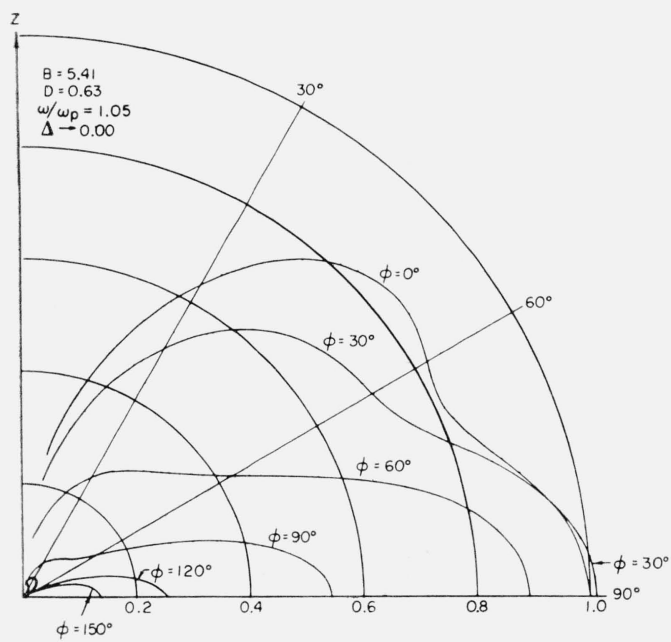

Figure 5. Normalized $\left|\mathrm{E}_{\phi}\right|$ versus $\theta$. (Norm. Const. $=10.01$ )

most easily in the broadside direction. In the vicinity of plasma resonance (fig. 7) the vertical pattern bulges above (and below) the equatorial plane, leaving a pronounced minimum in the forward direction. This salient feature, which also resembles the slotted, plasma-clad plane result and lends itself to a leaky-wave interpretation, could be used to determine plasma resonance. Figure 8 indicates the effect of varying the plasma density upon the phase lag in the equatorial plane.
The effect of sheath thickness $D$ is illustrated in figures 9 through 16 . The equatorial-plane amplitude patterns in figures 9 through 12 indicate a relative enhancement of the rearward radiation as the sheath thickness increases. This phenomenon may be interpreted as a peripheral wave which becomes more effectively trapped by the increasingly thick sheath. Similarly, figures 13 through 16 indicate a decreasing phase lag with increasing sheath thickness. Since the wave number, $k$, of the plasma is less than the free-space wave number, $k_{0}$, increasing the relative effect of the sheath by increasing its thickness will decrease the phase lag of this peripheral wave. Figures 15 and 16 , for a large-radius cylinder $(B=10)$, indicate considerably greater phase lags than those for the smaller-radius cylinder $(B=5.41)$ of figures 13 and 14 . The path length traveled in the plasma by the trapped wave is greater for the cylinder of larger radius; consequently, the increased phase lag is expected.

Figures 17 and 18 illustrate the relatively small effect of the slot width upon the equatorial-plane amplitude patterns. Although at wide angles the pattern becomes somewhat flattened, the effect is not considerable. The largest slot investigated, $\Delta=0.30$, corresponds to an opening of 22.6 degrees. Even for this relatively wide slot the pattern is not significantly different. Consequently, a justifiable simplification of the formulas would be the assumption of an infinitely thin slot.

An extensive computer program is necessary to calculate the exact equatorial plane fields without making the thin sheath approximations [Rotman 


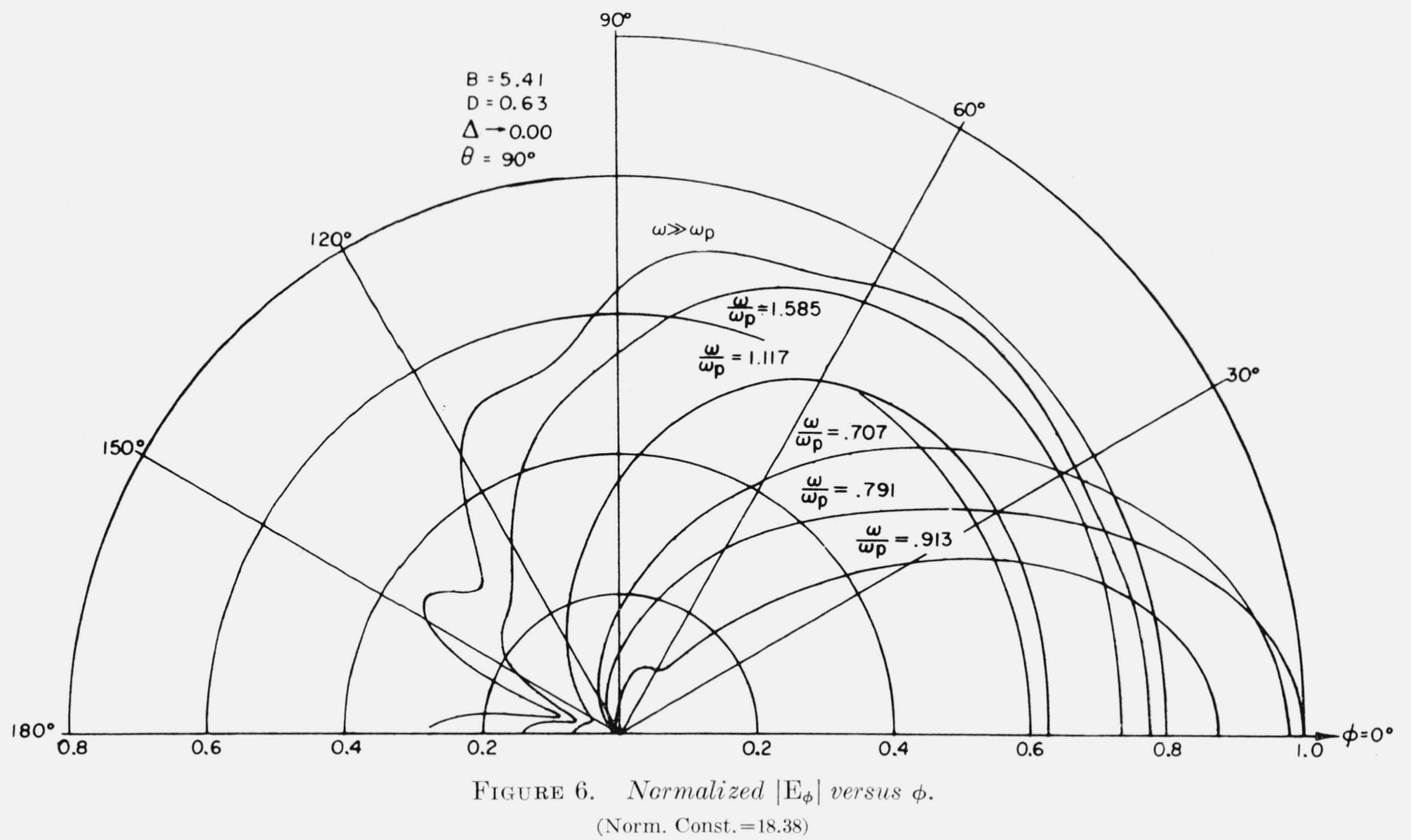

and Meltz, 1961]. A comparison of the exact results with the equatorial plane thin sheath approximations is presented in figure 19. With a sheath thickness of about 0.03 wavelength it is apparent that the difference is negligible. However, for a sheath thickness of about 0.5 wavelength (fig. 20) the difference is appreciable, although the qualitative features of the two patterns are approximately the same.

\section{Discussion}

Equation (5) presents a useful expression for the radiation field of an axially-slotted plasma-clad cylinder. It may be used to investigate the various parameter dependences of the system, and to predict its radiation properties. The expression, valid only for relatively thin sheaths, may be used for lossy plasmas without introducing serious analytical difficulties. The thin sheath approximation eliminates the possibility of cylindrical functions with imaginary or complex arguments which occur beyond certain critical angles and below plasma resonance. It is to be expected that under certain conditions these results may be applied to slotted, plasma-clad cylinders of finite length.

The numerical results clearly indicate a modification of the radiation pattern by the presence of the plasma sheath, the greatest changes occurring near or below the plasma-resonance frequency. Many of the properties may be understood in terms of a trapped peripheral wave, although this interpretation is not as evident below the plasma resonance. Several of the pattern characteristics may be used as plasma diagnostics, e.g., figure 7.

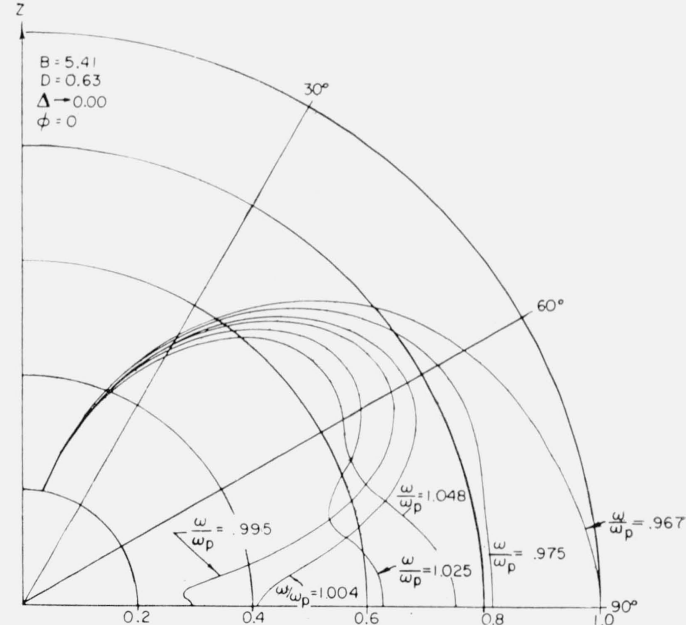

Figure 7. Normalized $\left|\mathrm{E}_{\phi}\right|$ versus $\boldsymbol{\theta}$.

(Norm. Const. $=12.94$ )

An important aspect of this radiating system requiring further investigation is the variation of slot impedance with plasma density. The numerical results of this report assumed constant slot voltage. However, for a more realizable driving source, the relative amplitudes of the patterns would be considerably modified; in particular, it is expected that the relative amplitudes below plasma resonance would be greatly reduced. 


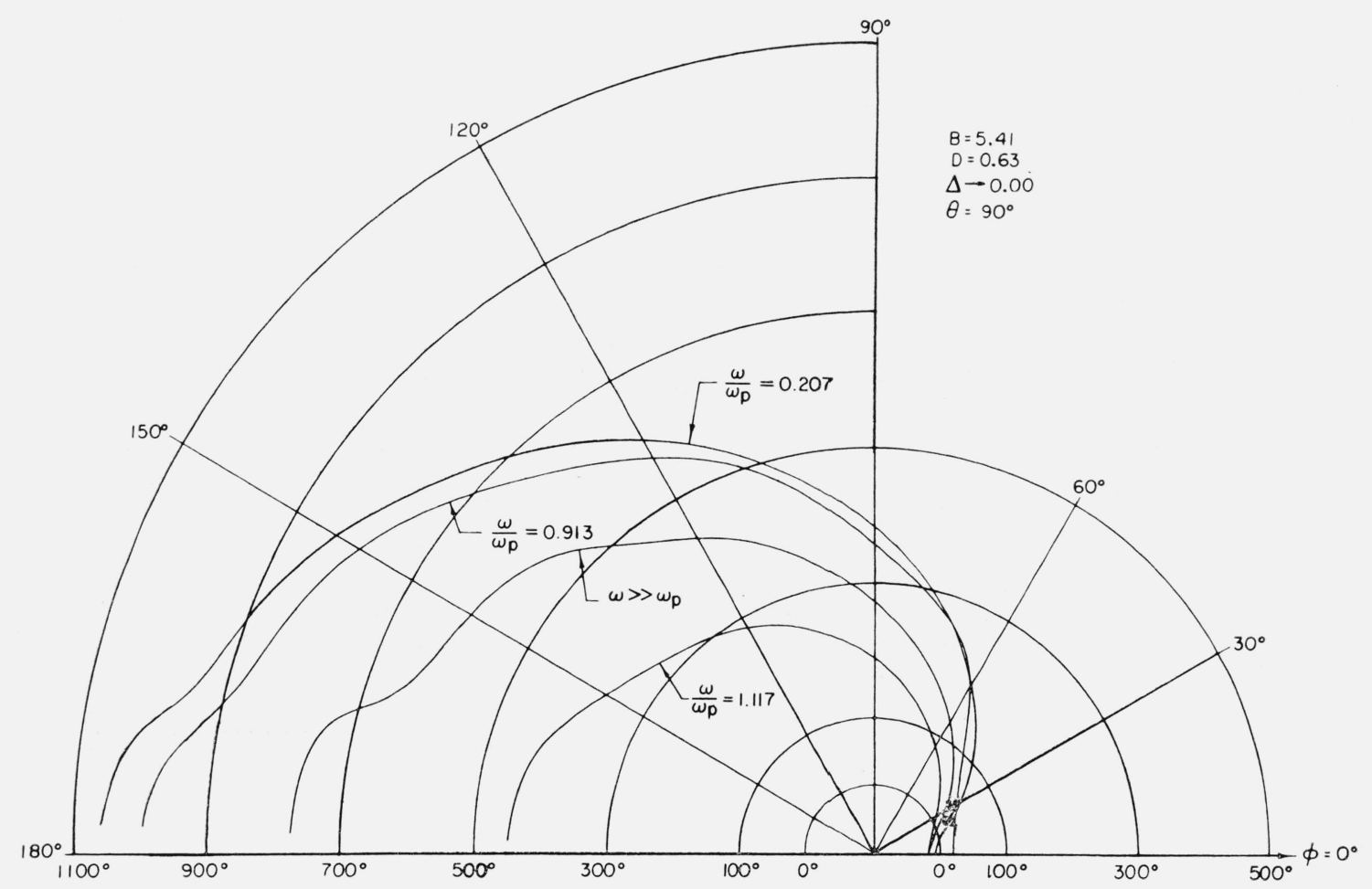

Figure 8. Phase lag of $\mathrm{E}_{\phi}$ in degrees versus $\phi$.

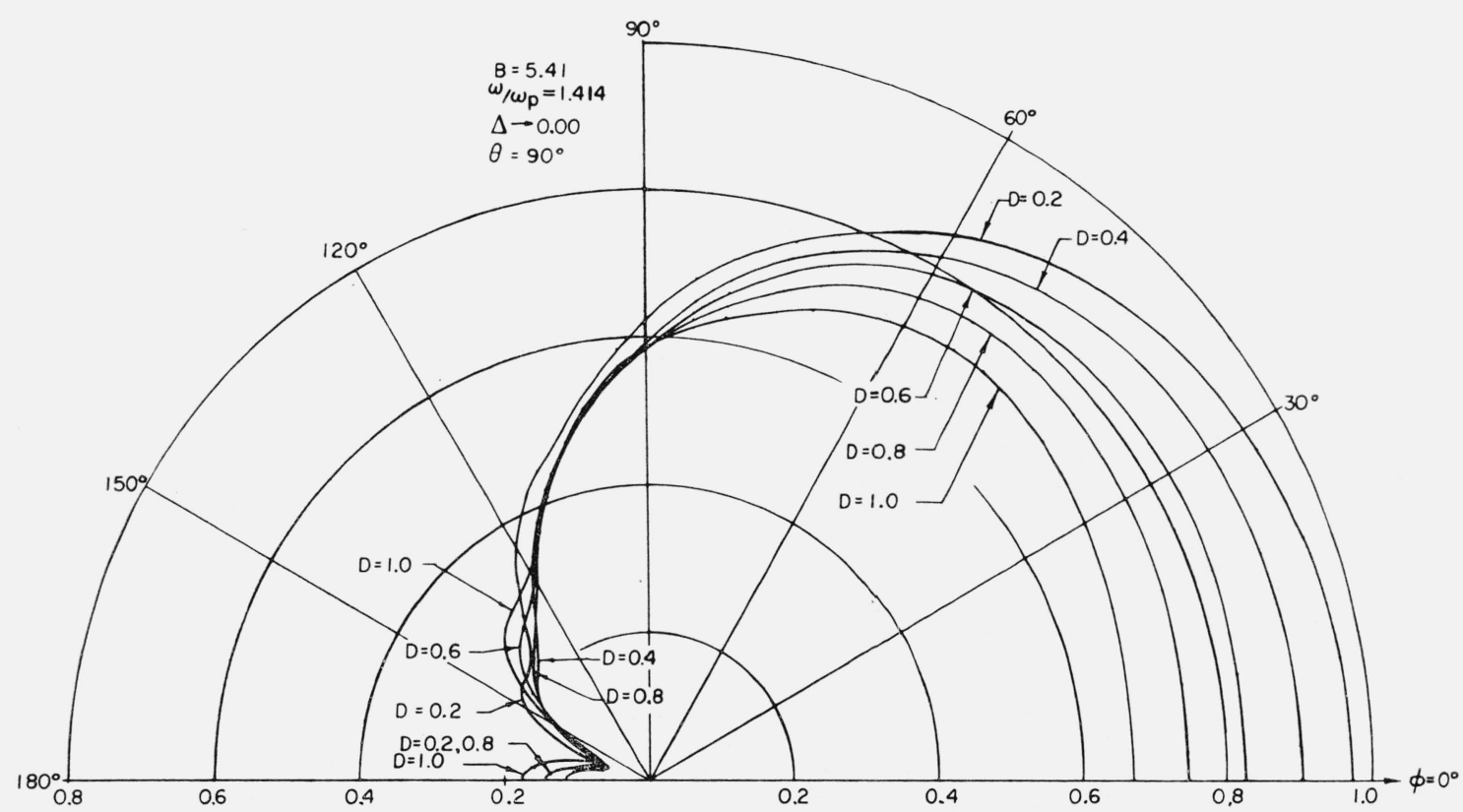

Figure 9. Normalized $\left|\mathrm{E}_{\phi}\right|$ versus $\phi$.

(Norm. Const. $=16.33$ ) 


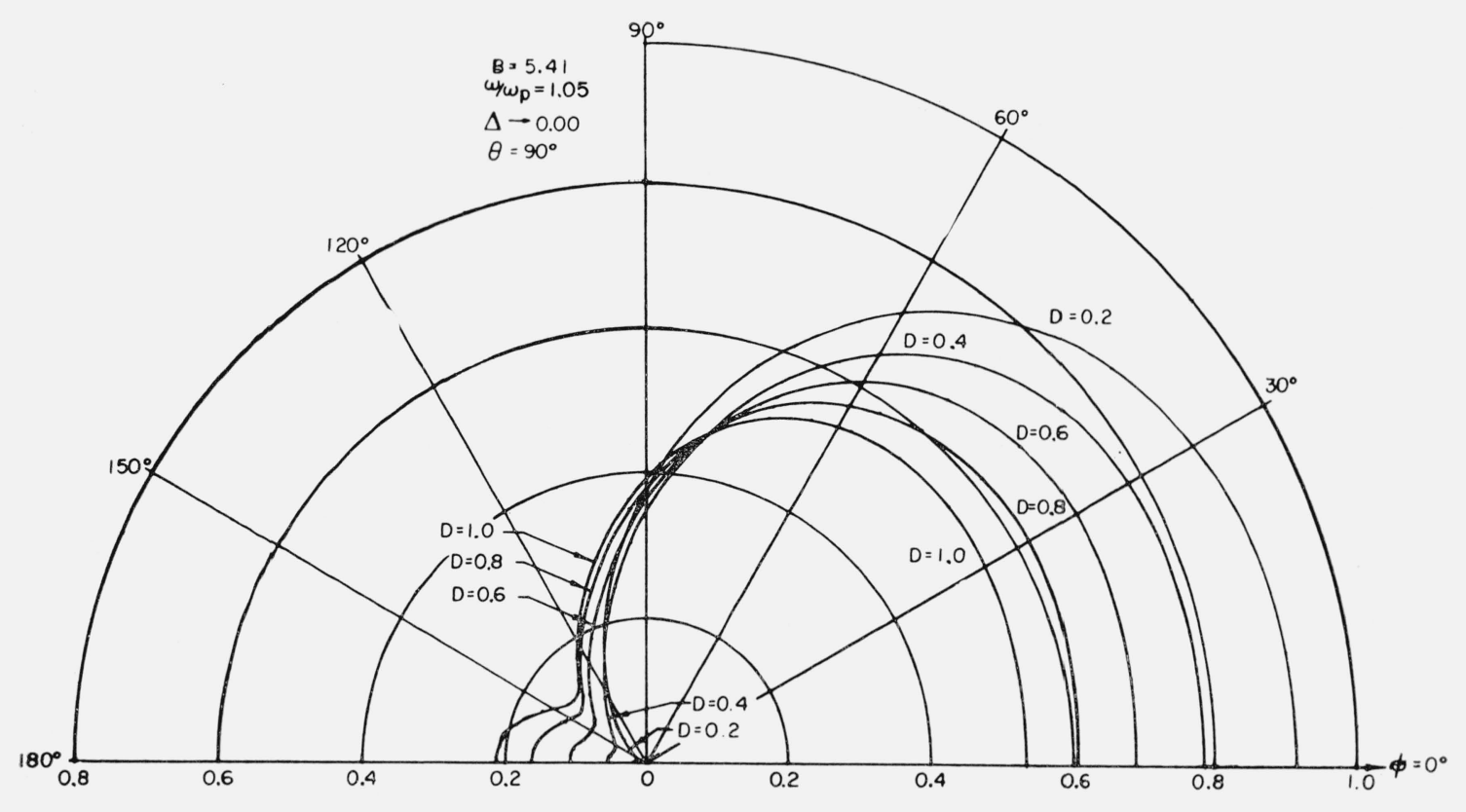

Figure 10. Normalized $\left|\mathrm{E}_{\phi}\right|$ versus $\phi$. (Norm. Const. $=14.8$ )

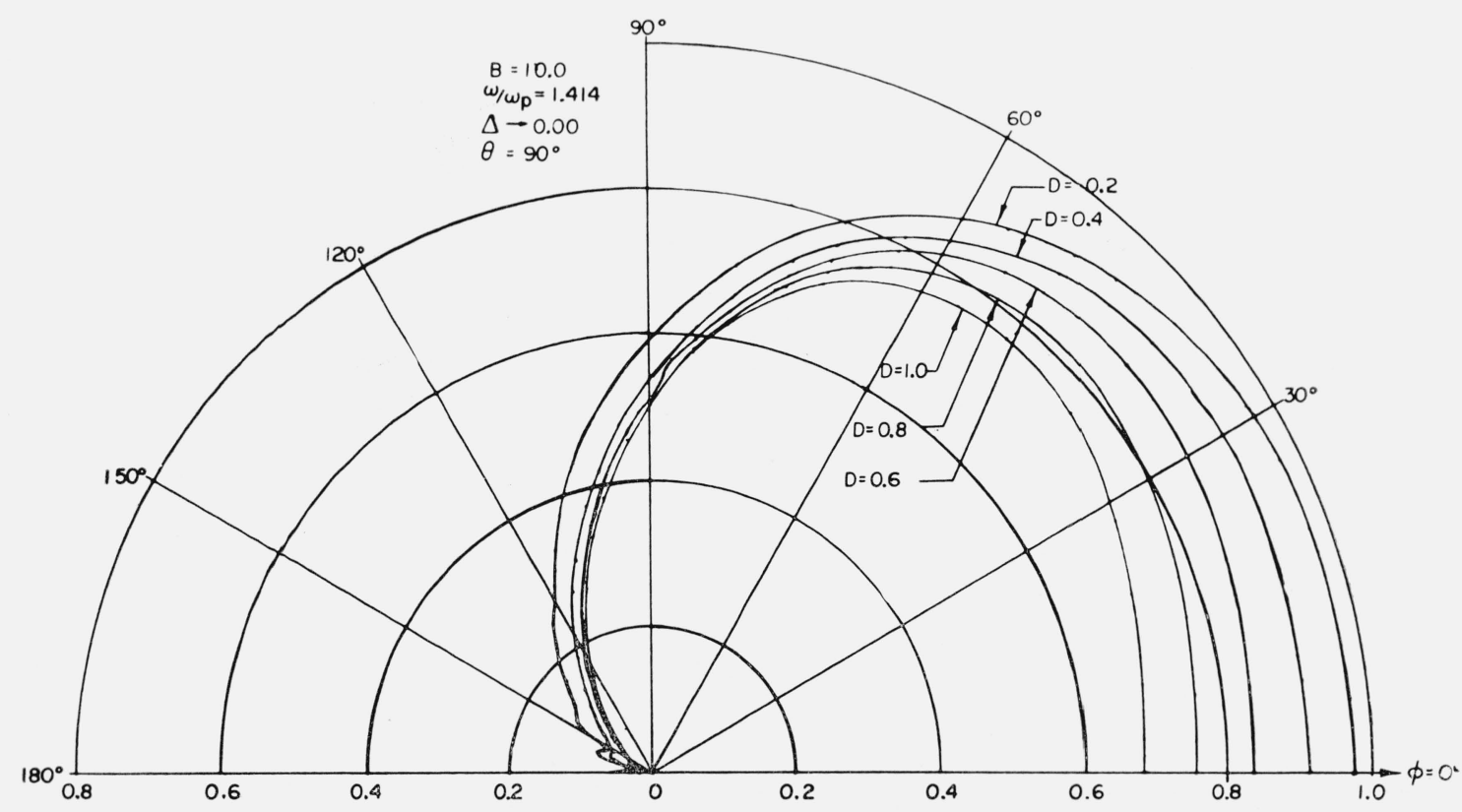

Figure 11. Normalized $\left|\mathrm{E}_{\phi}\right|$ versus $\phi$. (Norm. Const. $=30.7$ ) 


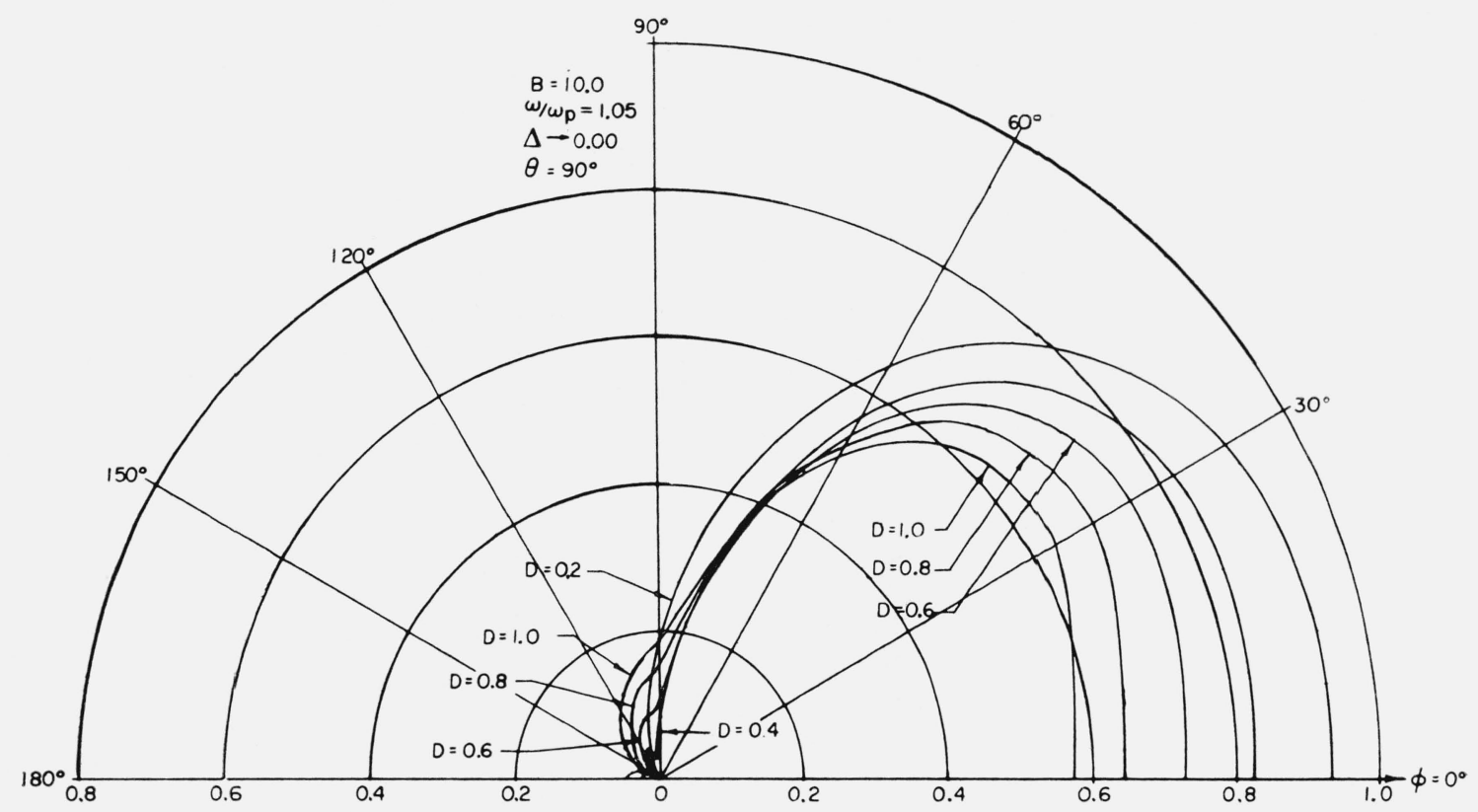

Figure 12. Normalized $\left|\mathrm{E}_{\phi}\right|$ versus $\phi$.

(Norm. Const. $=28.89)$

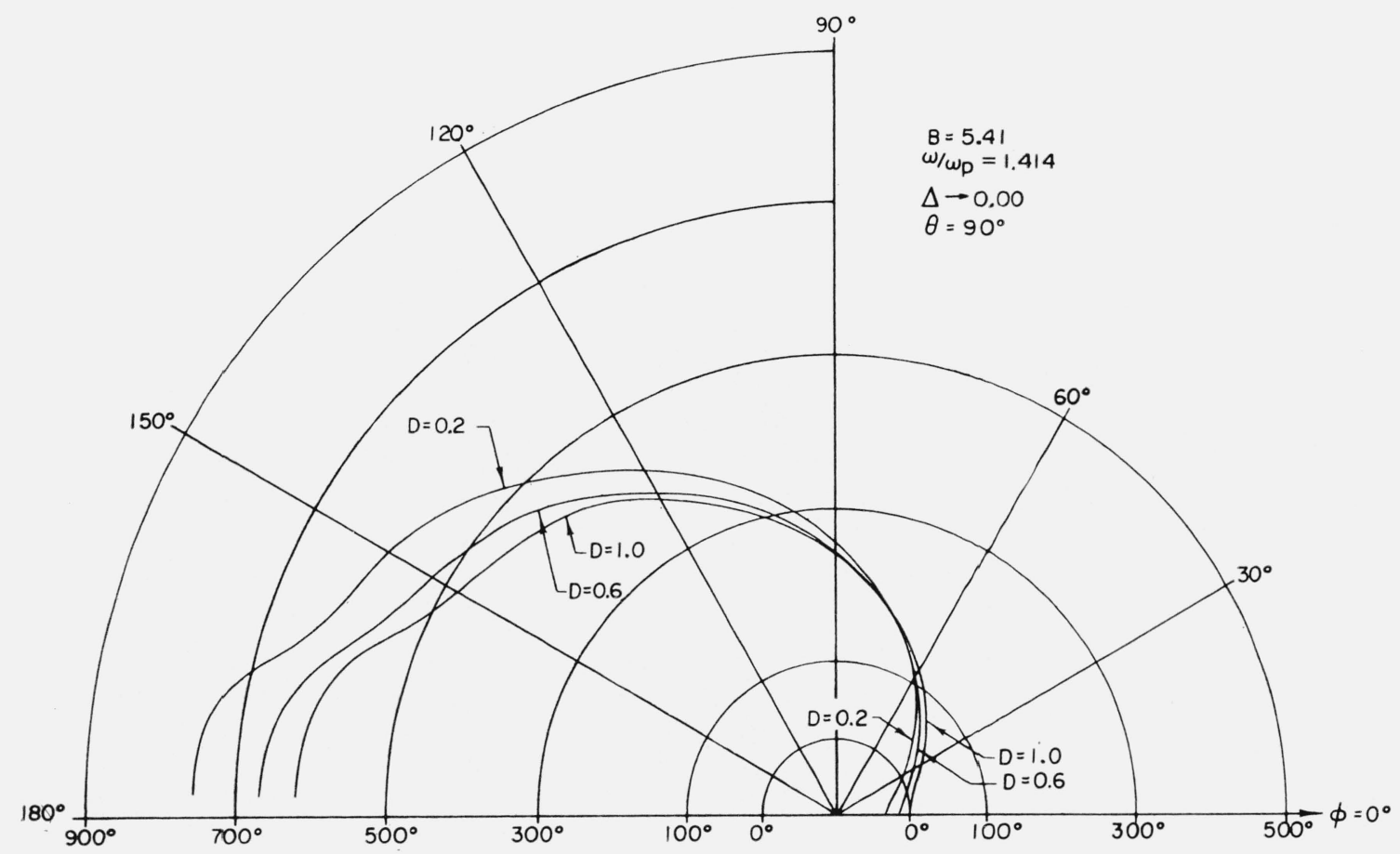

Figure 13. Phase lag of $\mathrm{E}_{\phi}$ in degrees versus $\phi$. 


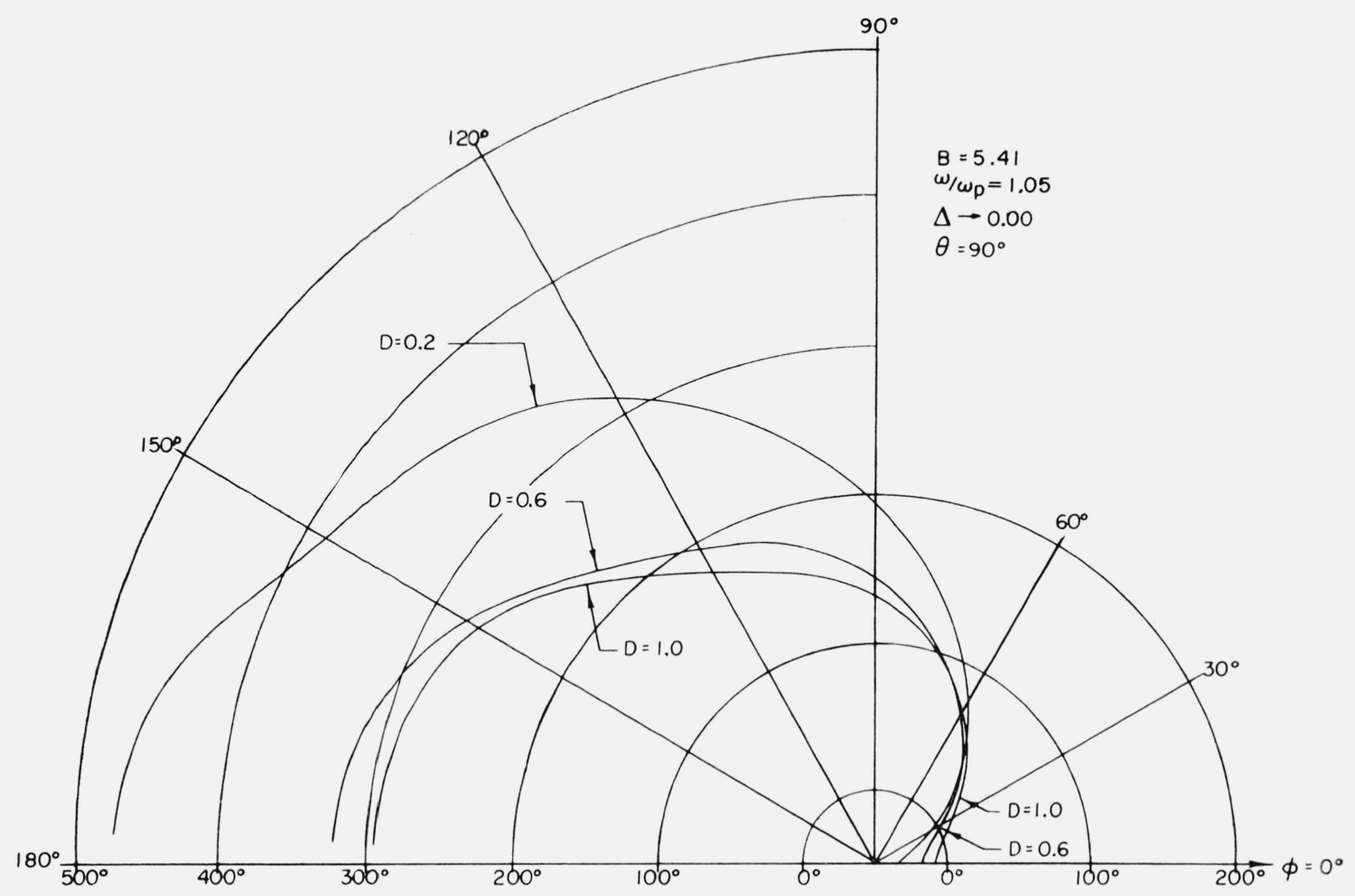

Figure 14. Phase lag of $\mathrm{E}_{\phi}$ in degrees versus $\phi$.

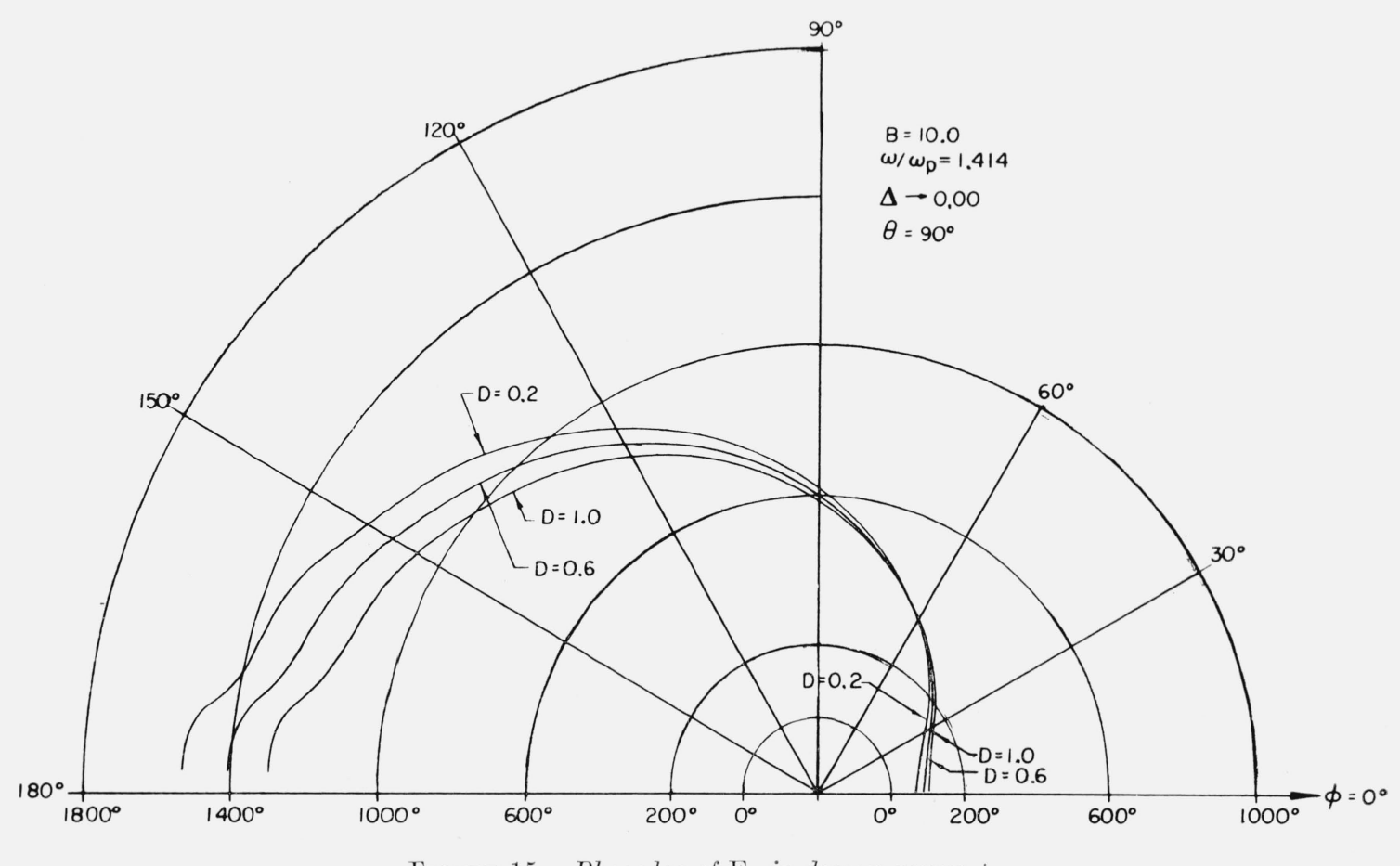

Figure 15. Phase lag of $\mathrm{E}_{\phi}$ in degrees versus $\phi$. 


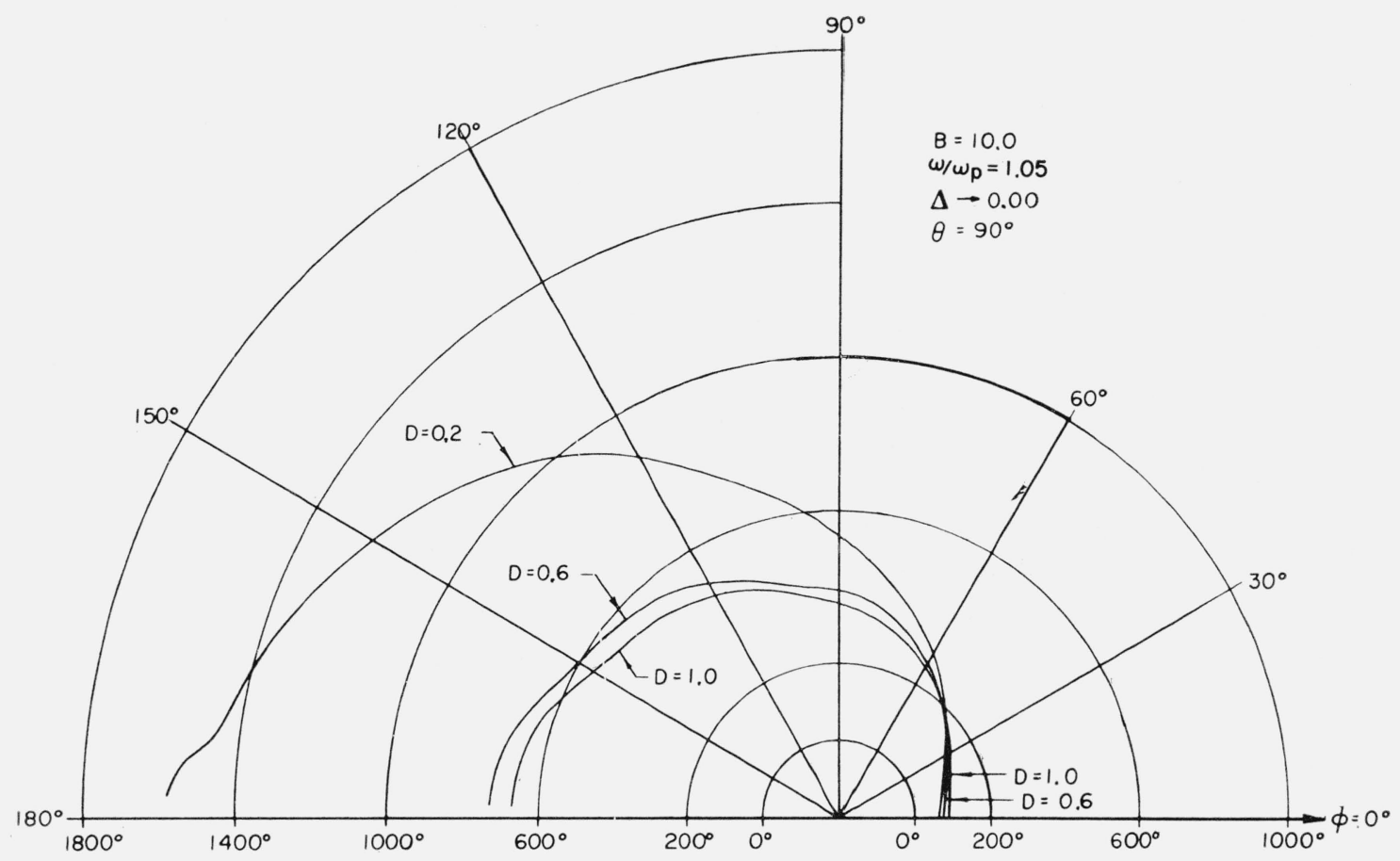

Figure 16. Phase lag of $\mathrm{E}_{\phi}$ in degrees versus $\phi$.

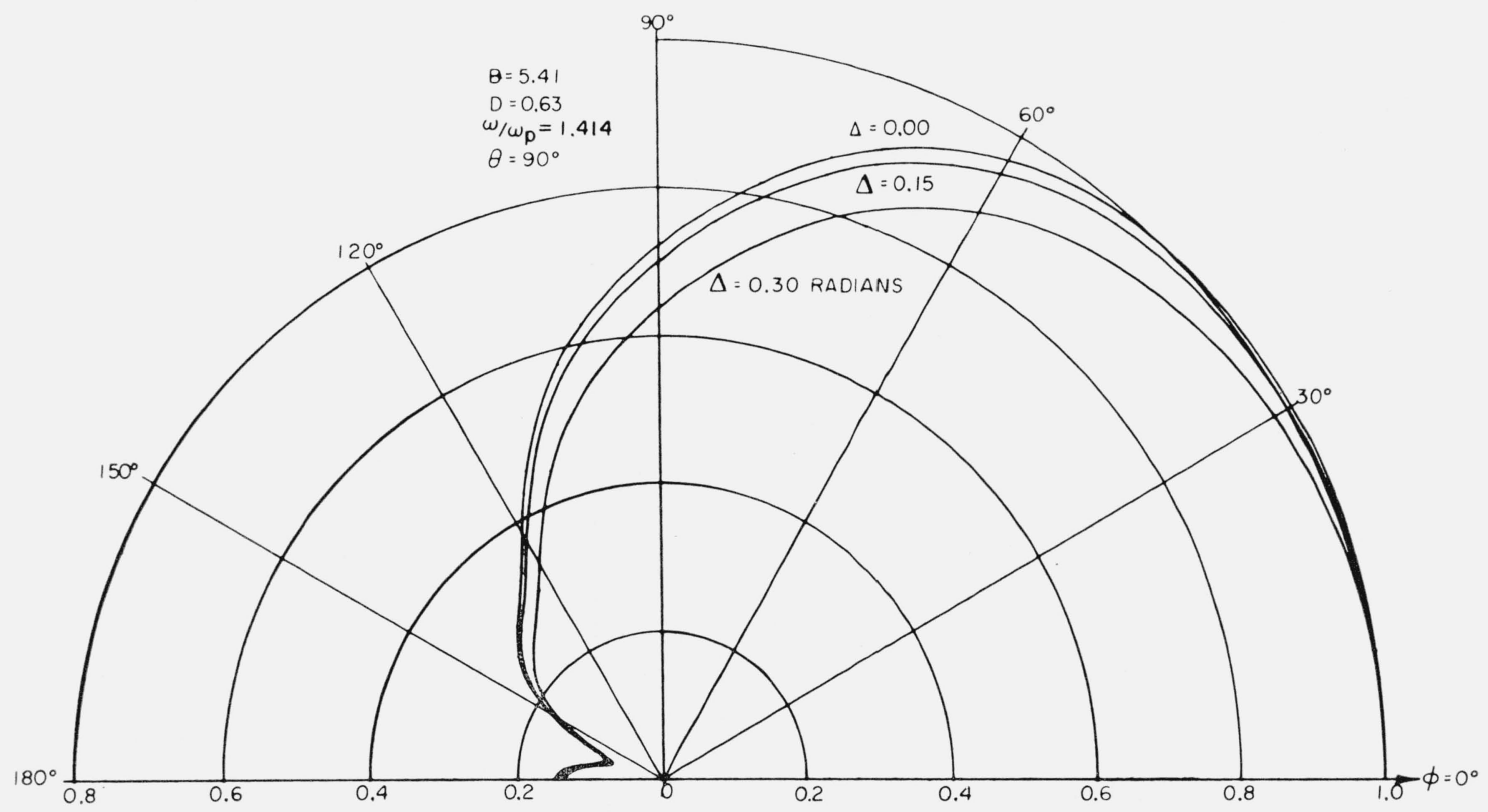

Figure 17. Normalized $\left|\mathrm{E}_{\phi}\right|$ versus $\phi$.

(Norm. Const. $=13.26$ ) 


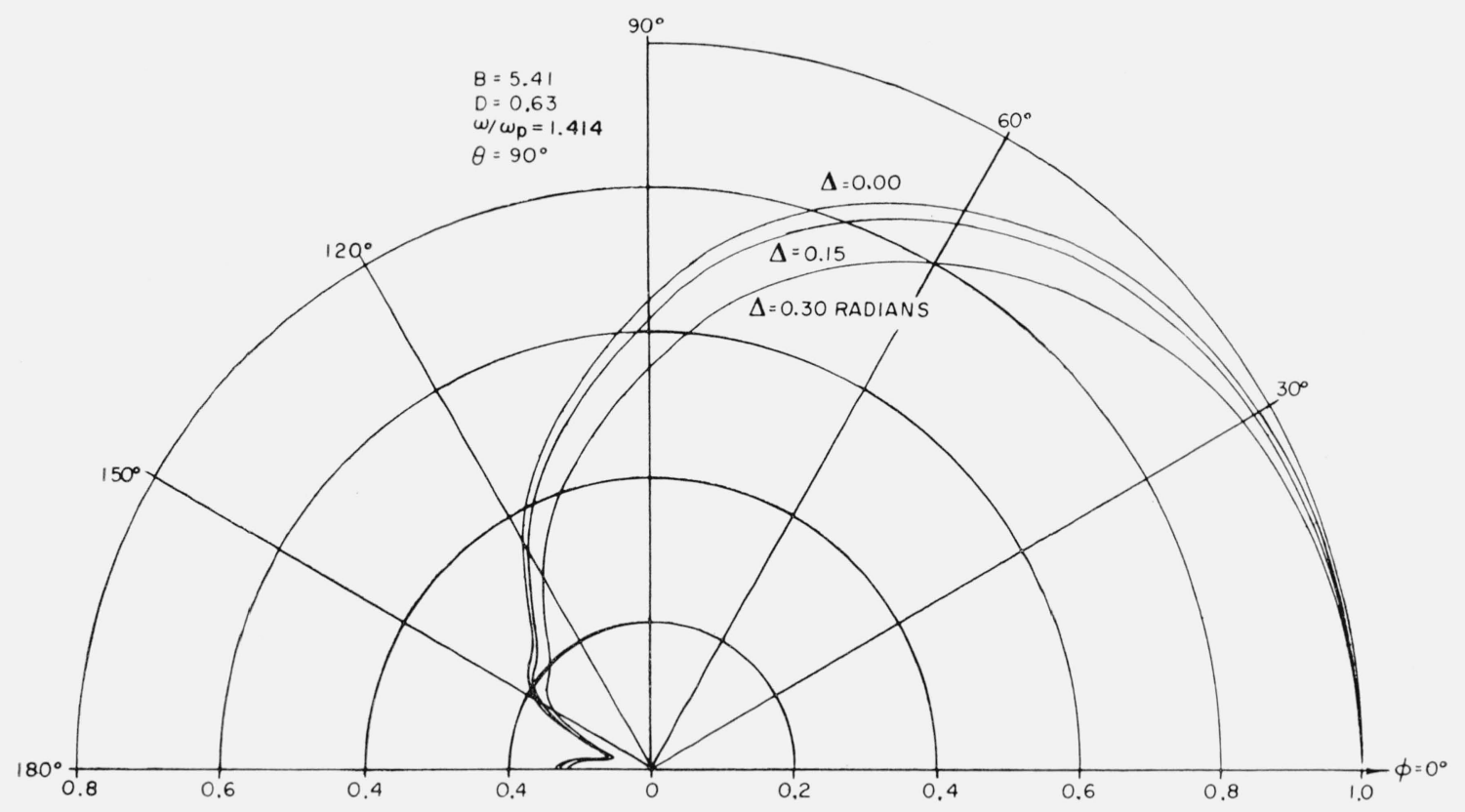

Figure 18. Normalized $\left|\mathrm{E}_{\phi}\right|$ versus $\phi$.

(Norm. Const. $=15.28$ )

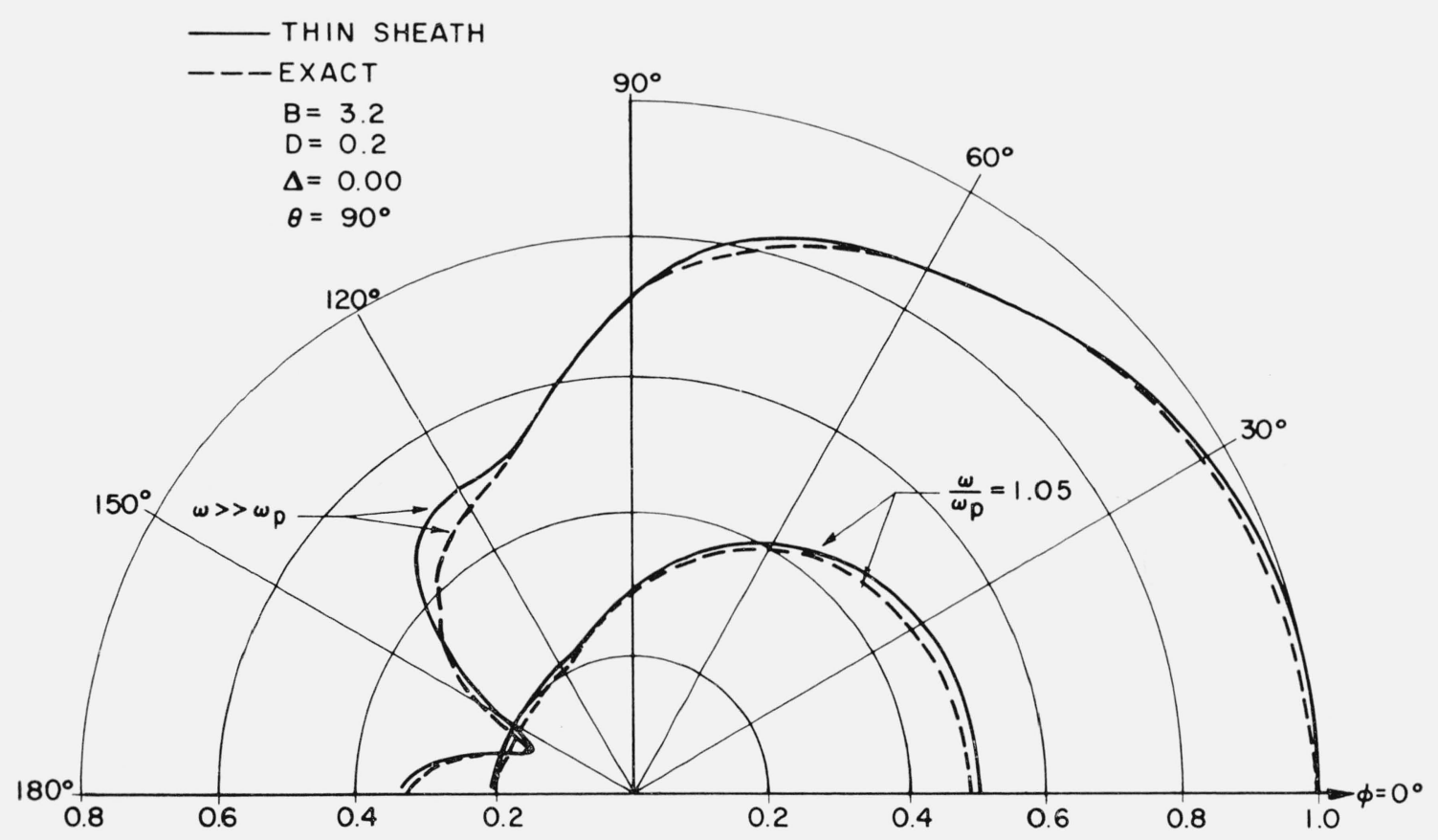

FiguRE 19. Normalized $\left|\mathrm{E}_{\phi}\right|$ versus $\phi$ (comparison of thin sheath approximation with exact results). 


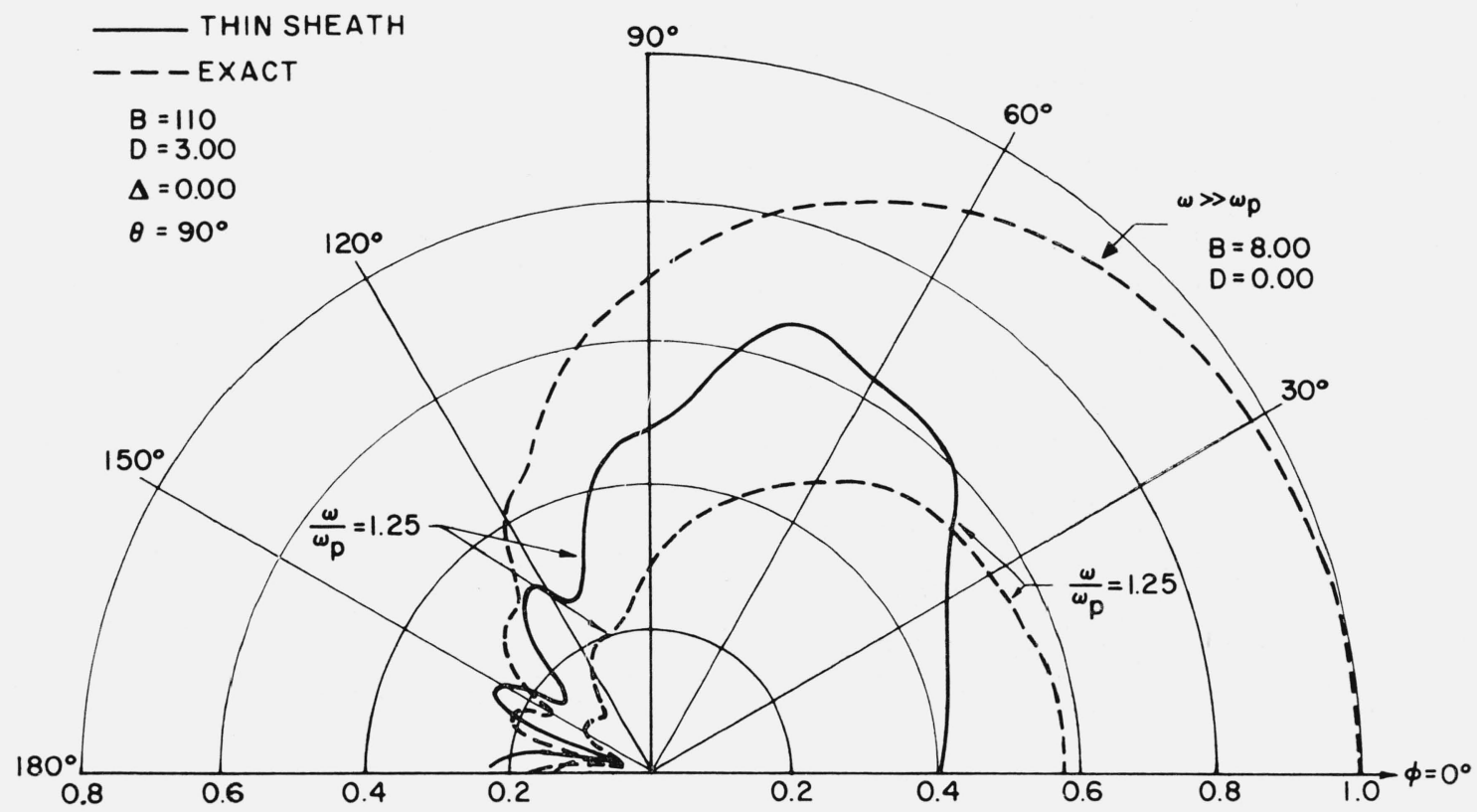

Figure 20. Normalized $\left|\mathrm{E}_{\phi}\right|$ versus $\phi$ (comparison of thin sheath approximation with exact results).

The author acknowledges, with thanks, the efforts of Cleyon Yowell in checking the calculations, Ray Stone in preparing the computer program, and David Nakatani in plotting the curves.

The calculations were carried out on the IBM 7090 Computer of the Western Data Processing Center, U.C.L.A.

\section{References}

Rotman, W., and G. Meltz (March 1961), Experimental investigation of the electromagnetic effects of reentry, AFCRL 87.

Stratton, J. A. (1941), Electromagnetic theory. Chapter VI, pp. 349-391. (McGraw-Hill Book Company, Inc., New York, N.Y.)

Wait, J. R. (1959), Electromagnetic radiation from cylindrical structures. Ch. 16, pp. 125-141. (Pergamon Press, New York, N.Y.) 